\title{
Caracterização da participação social de indivíduos na fase crônica pós-acidente vascular encefálico
}

\section{Social participation of individuals with chronic stroke}

\author{
Iza de Faria-Fortini ${ }^{1}$, Marluce Lopes Basílio ${ }^{1}$, Janaine Cunha Polese ${ }^{1}$, \\ Kênia Kiefer Parreira de Menezes ${ }^{1}$, Christina DCM Faria ${ }^{1}$, \\ Aline Alvim Scianni ${ }^{1}$, Luci Fuscaldi Teixeira-Salmela ${ }^{1}$
}

http://dx.doi.org/10.11606/issn.2238-6149.v28i1p71-8

\begin{abstract}
Faria-Fortini I, Basílio ML, Polese JC, Menezes KKP, Faria CDCM, Scianni AA, Teixeira-Salmela LF. Caracterização da participação social de indivíduos na fase crônica pós-acidente vascular encefálico. Rev Ter Ocup Univ São Paulo. 2017 jan.-abr.;28(1):71-8.
\end{abstract}

RESUMO: O objetivo foi descrever e comparar diferenças na restrição na participação social de indivíduos após acidente vascular encefálico (AVE) na fase crônica, considerando as deficiências, limitações em mobilidade e fatores pessoais. A restrição na participação social foi mensurada pelo LIFE-H 3.1-Brasil; as deficiências pela Escala de Depressão Geriátrica e Escala de FuglMeyer (EFM); as limitações em mobilidade pela velocidade de marcha e Timed Up \& Go (TUG) e os fatores pessoais incluíram sexo, idade, situação familiar e tempo de evolução pós-AVE. Observou-se restrição significativa na área de recreação. Em geral, restrições na participação social foram observadas em participantes com escores $\leq 28$ na EFM-membro inferior, sintomas de depressão, marcha comunitária limitada, risco de quedas e tempo de evolução $\leq 5$ anos. Aqueles com escores $\leq 45$ na EFM-membro superior apresentaram maior restrição em algumas áreas das atividades diárias. As mulheres apresentaram maior restrição em papéis sociais. Os resultados sugerem que deficiências e limitações relacionadas aos membros inferiores, sintomas depressivos e tempo de evolução devem ser considerados na avaliação, quando o objetivo da reabilitação for aumentar a participação social de indivíduos pós-AVE.

DESCRITORES: Participação social; Acidente vascular cerebral; Classificação Internacional de Funcionalidade Incapacidade e Saúde.
Faria-Fortini I, Basílio ML, Polese JC, Menezes KKP, Faria CDCM, Scianni AA, Teixeira-Salmela LF. Social participation of individuals with chronic stroke. Rev Ter Ocup Univ São Paulo. 2017 Jan.-Apr.;28(1):71-8.

ABSTRACT: This study aimed to describe and compare differences in restrictions in social participation of chronic stroke subjects, considering the impairments, mobility limitations, and personal factors. Restrictions in social participation was measured by the LIFE-H 3.1- Brasil; impairments by the Geriatric Depression Scale and FuglMeyer Assessment (FMA); mobility limitations by gait speed and Timed Up \& Go (TUG) test; whereas personal factors included sex, age, family status, and time after stroke onset. Significant restrictions in the recreation area were found. In general, restrictions in social participation were observed in participants with scores $\leq 28$ at the FMA-lower extremity section, depressive symptoms, limited-community ambulation status, risk of falls, and time after stroke $\leq 5$ years. Those with scores $\leq 45$ FMA-upper extremity showed greater restrictions in some areas of daily activities. Women showed greater restrictions in the social roles. The results suggested that impairments and limitations related to the lower extremity, depressive symptoms, and time after stroke onset should be considered for the assessment of stroke subjects, when the rehabilitation goal is to increase social participation.

KEYWORDS: Social participation; Stroke; International Classification of Functioning, Disability and Health.

Artigo originado de tese de Doutorado pelo Programa de Pós-Graduação em Ciências da Reabilitação da Universidade Federal de Minas Gerais. Pôster apresentado no X Congresso Brasileiro de Doenças Cerebrovasculares - AVC, Belo Horizonte, MG, 15-17 out. 2015 . Fonte de auxílio: CAPES.

1. NeuroGroup; Departamento de Fisioterapia, Escola de Educação Física, Fisioterapia e Terapia Ocupacional da Universidade

Federal de Minas Gerais (UFMG). E-mails: izafaria@yahoo.com.br, marlucelb@yahoo.com.br, janainepolese@yahoo.com.br, keniakiefer@yahoo.com.br, chrismoraisf@yahoo.com, ascianni@task.com.br, lfts@ufmg.br

Endereço para correspondência: Prof ${ }^{a}$ Luci Fuscaldi Teixeira-Salmela - Departamento de Fisioterapia, Universidade Federal de Minas Gerais - Campus Pampulha. Avenida Antônio Carlos, 6627. Belo Horizonte, MG, Brasil. CEP: 31270-901. E-mail: 1fts@ufmg.br 


\section{INTRODUÇÃO}

$\mathrm{O}$ Acidente Vascular Encefálico (AVE) representa a principal causa de morte e incapacidade no Brasil, com relevante impacto econômico e social ${ }^{1}$. A restrição na participação social é comumente observada em indivíduos com condições crônicas, como o AVE, sendo que 65\% reportam restrição na reintegração em atividades na comunidade e no papel social e mais de $50 \%$ apresentam comprometimento funcional, de tal forma que precisam de algum tipo de ajuda externa para realizar as atividades cotidianas $^{2}$.

Segundo a Classificação Internacional de Funcionalidade, Incapacidade e Saúde (CIF), a restrição na participação é resultante da interação entre aspectos referentes à deficiência na estrutura e função do corpo, limitação em atividades, fatores contextuais, o que inclui fatores ambientais e pessoais, e também da própria condição de saúde ${ }^{3}$. Após a ocorrência do AVE, muitos sobreviventes apresentam algum grau de deficiência na função motora ${ }^{4}$, consideráveis limitações na marcha ${ }^{5}$ e elevada preocupação com a ocorrência de quedas ${ }^{6}$, aspectos estes que podem comprometer a participação social. Adicionalmente, a ocorrência de sintomas depressivos e fatores pessoais, tais como sexo, idade e situação familiar também estão associados à restrição na participação social ${ }^{7}$. Outro fator pessoal que pode ser considerado é o tempo de evolução após o $\mathrm{AVE}^{8}$. Ainda não foi reportado na literatura a existência de associação dessa variável com restrição na participação social, no entanto, estudo prévio demonstrou declínio funcional em indivíduos pós-AVE com o passar dos anos, sendo esse declínio independente de fatores como idade, gravidade e recorrência do AVE' .

A caracterização da participação social após o AVE pode demonstrar o grau de envolvimento dos indivíduos nas situações de vida cotidiana. Além disso, a análise de outros componentes da funcionalidade e fatores contextuais pode ser importante na identificação dos aspectos que mais contribuem para a restrição na participação social. Desta forma, os objetivos deste estudo foram descrever e comparar diferenças na restrição na participação social de indivíduos após o AVE na fase crônica, considerando as deficiências em função e estrutura do corpo (sintomas depressivos e função motora de membros superior e inferior), limitação em mobilidade (velocidade de marcha e riscos de queda) e fatores pessoais (idade, sexo, situação familiar e tempo de evolução pós-AVE).

\section{MÉTODO}

\section{Participantes}

Estudo observacional exploratório, conduzido com indivíduos pós-AVE, residentes na comunidade. Indivíduos com diagnóstico de AVE foram recrutados na comunidade e em serviços públicos de reabilitação, entre março de 2013 a agosto de 2014, considerando os seguintes critérios de inclusão: idade $\geq 20$ anos; diagnóstico de AVE unilateral, com no mínimo seis meses de evolução; hemiparesia caracterizada pelo aumento de tônus dos flexores de cotovelo e/ou extensores do joelho (escore diferente de zero na Escala Modificada de Ashworth) ou fraqueza muscular de preensão manual e/ou extensores de joelho (diferença superior a $10 \%$ e $15 \%$, respectivamente, entre as medidas dos lados parético e não parético); e ausência de déficits cognitivos avaliados pelo Mini-Exame do Estado Mental (pontos de corte 13 para indivíduos analfabetos, 18 para baixa e média escolaridade e 26 para alta escolaridade ${ }^{10}$. Foram excluídos indivíduos que apresentassem afasia motora que os impossibilitasse de responder perguntas verbalmente, ou demais condições de saúde não relacionadas ao AVE.

\section{Aspectos éticos}

Este estudo foi aprovado pelos Comitês de Ética em Pesquisa da Universidade Federal de Minas Gerais e da Secretaria Municipal de Saúde da Prefeitura de Belo Horizonte (pareceres 113.846/2012 e 326.216/2013). Todos os participantes foram informados sobre os objetivos do estudo e os procedimentos aos quais seriam submetidos e assinaram o Termo de Consentimento Livre e Esclarecido.

\section{Procedimentos}

Após verificação dos critérios de elegibilidade, os participantes foram avaliados nas dependências do Departamento de Fisioterapia da Universidade Federal de Minas Gerais e em Centros de Reabilitação da rede do Sistema Único de Saúde da Prefeitura Municipal de Belo Horizonte por cinco examinadores previamente treinados na aplicação dos testes e que possuíam pelo menos cinco anos de experiência clínica e/ou em pesquisa na área. Os questionários foram aplicados por meio de entrevista, a fim de evitar erros de interpretação, já que alguns indivíduos eram analfabetos ou analfabetos funcionais. 


\section{Medidas de avaliação}

\section{Restrição na participação social}

A restrição na participação social foi mensurada por meio da aplicação da versão adaptada do Assesment of Life Habits 3.1 (LIFE-H 3.1- Brasil) ${ }^{11}$. Este questionário é composto por 77 questões agrupadas em duas subescalas: atividades diárias, composto pelas áreas nutrição, condicionamento físico, cuidado pessoal, comunicação, moradia e mobilidade; e papéis sociais, que inclui as áreas responsabilidade, relações interpessoais, vida em comunidade, educação, emprego e recreação. Neste estudo, a área educação não foi incluída nas análises, uma vez que a média de idade da amostra foi de 58 anos e esta não é uma atividade cotidiana nesta faixa etária. A avaliação do desempenho identifica, em cada uma das questões, o nível de realização de cada atividade (sem dificuldade, dificuldade, realizado por um responsável, não realizado, ou não se aplica) e do tipo de assistência requerida (sem assistência, dispositivo de auxílio, adaptação e assistência humana $)^{11}$. A partir da combinação do nível de realização e do tipo de assistência, o desempenho é classificado em uma escala de zero a nove, onde zero indica total restrição e nove ausência de restrição na participação social, sendo a atividade diária ou papel social realizado sem dificuldade ou assistência. $\mathrm{O}$ escore total, por subescala e por área é obtido pela fórmula: ( $\Sigma$ pontuações*10)/(número de itens aplicáveis*9), sendo que zero indica total restrição na participação social e 10 nenhuma restrição ${ }^{11}$. O LIFE-H 3.1-Brasil apresentou adequada validade e confiabilidade dos indivíduos $(0,90-0,91)$ e dos itens $(0,97-0,98)$ em indivíduos pós-AVE ${ }^{11}$.

\section{Deficiências em estrutura e função do corpo}

A versão adaptada da Escala de Depressão Geriátrica (EDG) foi utilizada para determinação de sintomas depressivos. Neste estudo, foi utilizada a versão reduzida com 15 itens, que apresenta adequada confiabilidade e validade para rastreamento de quadros depressivos ${ }^{12}$. O ponto de corte $\geq 6$ pontos na EDG foi utilizado para determinar a presença de sintomas depressivos ${ }^{13}$.

A função motora dos membros superior e inferior paréticos foi avaliada através da aplicação da versão brasileira da Escala de Fugl-Meyer (EFM), específica para mensuração da função sensório motora em indivíduos pós-AVE, que apresenta adequada confiabilidade $^{14}$. É destinado um total de 100 pontos para a função motora normal, sendo que a pontuação máxima do membro inferior é 34 pontos e a do membro superior é 66 pontos $^{14}$. Escores $\leq 28$ pontos indicam deficiência da função motora de membro inferior ${ }^{15}$, enquanto escores $\leq 45$ pontos, deficiência da função motora de membro superior ${ }^{16}$.

\section{Limitações em mobilidade}

Para avaliação da limitação de atividades, foi considerado o domínio de mobilidade da CIF. Para avaliação da velocidade de marcha, os indivíduos foram orientados a deambular na velocidade habitual ao longo de uma superfície plana de 14 metros de extensão. O período de aceleração e desaceleração, correspondente aos dois primeiros e dois últimos metros, não foi considerado. Portanto, foi medido o tempo necessário para percorrer os 10 metros centrais. A velocidade de marcha foi expressa em metros por segundo $(\mathrm{m} / \mathrm{s})^{17}$. Considera-se marcha comunitária limitada quando a velocidade for inferior a $0,8 \mathrm{~m} / \mathrm{s}^{18}$. Este teste apresenta adequados valores de confiabilidade e resultados consistentes em apenas uma única aplicação, em indivíduos pós-AVE ${ }^{17}$.

$\mathrm{O}$ teste Timed Up and Go (TUG) mensura a mobilidade básica, o que inclui habilidade para levantar de uma cadeira, andar uma distância de três metros, girar, andar de volta para a cadeira e sentar-se novamente ${ }^{17}$. A limitação na mobilidade é frequentemente relacionada a um maior risco de quedas, sendo o TUG comumente utilizado para rastreio do risco de quedas ${ }^{19}$. Persson et al. ${ }^{19}$ concluíram que indivíduos pós-AVE, com escores $\geq 15$ segundos no TUG, apresentaram alto risco de risco de quedas. No presente estudo, este foi o ponto de corte considerado. Este teste apresenta adequada confiabilidade para aplicação em indivíduos pós-AVE e escores consistentes em apenas uma repetição após familiarização ${ }^{17}$.

\section{Fatores pessoais}

Os dados referentes aos fatores pessoais (idade, sexo, situação familiar e tempo de evolução pós-AVE) foram coletados por meio de entrevista. Em relação à situação familiar, os indivíduos foram questionados se moravam sozinhos ou com companheiros e/ou familiares.

\section{Cálculo amostral}

O cálculo amostral foi baseado no número de variáveis utilizadas na comparação entre os grupos. Considerando as nove variáveis dependentes incluídas, como regra geral, seriam necessários pelo menos 10 
Faria-Fortini I, et al. Caracterização da participação social de indivíduos na fase crônica. Rev Ter Ocup Univ São Paulo. 2017 jan./abr.;28(1):71-8.

participantes por variável, o que corresponderia a uma amostra mínima de 90 participantes ${ }^{20}$.

\section{Análise dos dados}

Estatísticas descritivas foram utilizadas para descrição da restrição na participação social. Para caracterizar o nível de restrição na participação social, uma pontuação $\leq 5$ foi considerada como indicador de restrição significativa, uma vez que esse valor sinaliza que o hábito de vida foi realizado com assistência humana ou não foi mais realizado após o $\mathrm{AVE}^{21}$.

Para avaliar diferenças entre os grupos quanto ao nível de restrição na participação social, os indivíduos foram agrupados segundo as seguintes variáveis: deficiências (sintomas depressivos e função motora); limitação em mobilidade (velocidade de marcha e risco de queda) e fatores pessoais (idade, sexo, situação familiar e tempo de evolução). A normalidade dos dados foi verificada pelo teste de Kolmogorov-Smirnov. $\mathrm{Na}$ análise inferencial dos dados, utilizou-se o teste- $\mathrm{t}$ de Student para grupos independentes, para avaliar a diferença entre as médias dos escores do LIFE-H 3.1-Brasil (áreas, subescalas e total) para as seguintes variáveis: presença/ausência de sintomas depressivos $(\mathrm{EDG} \geq 6 /<6)^{13}$; presença/ausência de comprometimento motor de membro inferior $(E F M \leq 28 / E F M>28)^{15}$ e de membro superior $(\mathrm{EFM} \leq 45 / \mathrm{EFM}>45)^{16}$; marcha comunitária/marcha comunitária limitada (velocidade de marcha $>0,8 / \leq 0,8 \mathrm{~m} / \mathrm{s})^{18}$; presença/ausência de risco de quedas (TUG $\geq 15 /<15$ segundos) ${ }^{19}$; idade $(<60 / \geq 60$ anos); sexo (feminino/masculino); situação familiar (vive sozinho/vive com companheiro e/ou familiares) e tempo de evolução ( $<5$ anos $/ \geq 5$ anos). O nível de significância para todas análises inferenciais foi de $5 \%$. As análises foram realizadas no programa SPSS for Windows, versão 18.0.

\section{RESULTADOS}

Foram recrutados por contato telefônico 485 indivíduos, sendo que 122 compareceram para avaliação. Após a avaliação inicial, 13 foram excluídos, por não atenderem aos critérios de elegibilidade. Desta forma, 109 indivíduos participaram deste estudo. As características demográficas e clínicas são apresentadas na Tabela 1. No entanto, alguns dados de deficiências e limitação de mobilidade de alguns indivíduos ficaram incompletos, como reportado na Tabela 3 , mas isso não ultrapassou $3 \%$.
Tabela 1 - Características dos participantes

\begin{tabular}{|c|c|}
\hline Característica & $(n=109)$ \\
\hline Idade (anos), média \pm DP (amplitude) & $58 \pm 12(20-83)$ \\
\hline Sexo (\%), homens & $64(59)$ \\
\hline $\begin{array}{l}\text { Tempo de evolução (anos), média } \pm \text { DP } \\
\text { (amplitude) }\end{array}$ & $\begin{array}{c}5 \pm 5 \\
(0,5-32)\end{array}$ \\
\hline $\begin{array}{l}\text { Função motora - EFM membro } \\
\text { superior (0-66), média } \pm D P(\text { amplitude) }\end{array}$ & $\begin{array}{l}44 \pm 19 \\
(4-66)\end{array}$ \\
\hline $\begin{array}{l}\text { Função motora - EFM membro } \\
\text { inferior (0-34), média } \pm \text { DP (amplitude) }\end{array}$ & $\begin{array}{l}27 \pm 7 \\
(8-34)\end{array}$ \\
\hline $\begin{array}{l}\text { Velocidade de marcha }(\mathrm{m} / \mathrm{s}) \\
\text { média } \pm \mathrm{DP} \text { (amplitude) }\end{array}$ & $\begin{array}{c}0,82 \pm 0,33 \\
(0,12-1,54) \\
\end{array}$ \\
\hline $\begin{array}{l}\text { Escala de depressão geriátrica (0-15), } \\
\text { média } \pm \text { DP (amplitude) }\end{array}$ & $\begin{array}{c}6 \pm 4 \\
(0-13)\end{array}$ \\
\hline TUG (s), média \pm DP (amplitude) & $18 \pm 11(8-88)$ \\
\hline
\end{tabular}

Os dados referentes ao nível de restrição na participação social na amostra total, descritos por área, subescalas e escore total do LIFE-H 3.1-Brasil, são apresentados na Tabela 2. Observou-se restrição significativa na participação social (escore $\leq 5,0)^{21}$ apenas na área de recreação $(4,6 \pm 2,9)$.

Tabela 2 - Média \pm DP e amplitude (mínimo-máximo) dos escores por área, subescala e total do LIFE-H 3.1 Brasil $(n=109)$

\begin{tabular}{ll}
\hline LIFE-H 3.1-Brasil & $\mathbf{n}=\mathbf{1 0 9}$ \\
\hline Atividades diárias & $7,4 \pm 1,4(3,3-9,8)$ \\
\hline Nutrição & $7,4 \pm 1,9(2,7-10)$ \\
\hline Condicionamento físico & $8,1 \pm 1,8(3,0-10)$ \\
\hline Cuidados pessoais & $8,0 \pm 1,8(3,2-10)$ \\
\hline Comunicação & $8,0 \pm 1,5(2,7-10)$ \\
\hline Moradia & $6,8 \pm 2,0(1,8-10)$ \\
\hline Mobilidade & $5,5 \pm 2,4(1,3-10)$ \\
\hline Papéis sociais & $6,7 \pm 2,1(1,9-10)$ \\
\hline Responsabilidades & $7,5 \pm 2,2(1,1-10)$ \\
\hline Relacionamentos interpessoais & $8,9 \pm 1,7(2,0-10)$ \\
\hline Vida em comunidade & $6,6 \pm 3,0(0,16-10)$ \\
\hline Emprego & $6,3 \pm 3,6(0,1-10)$ \\
\hline Recreação* & $4,6 \pm 2,9(0-10)$ \\
\hline Total & $7,1 \pm 1,7(2,7-9,8)$ \\
\hline$*$ Restrição significativa (escore $<5,0)$ &
\end{tabular}

Os dados referentes às comparações do nível de restrição na participação social entre os grupos, de acordo com as deficiências, limitações em mobilidade e fatores pessoais, são apresentados nas Tabelas 3 e 4 . Quanto às variáveis de deficiências e limitações em mobilidade (Tabela 3), o grupo com presença de comprometimento motor de membro inferior $(\mathrm{EFM} \leq 28)^{15}$, sintomas depressivos $(E D G \geq 6)^{13}$, marcha comunitária limitada 
$(\leq 0,8 \mathrm{~m} / \mathrm{s})^{18}$ e riscos de queda $(\mathrm{TUG} \geq 15)^{19}$ apresentou maior restrição na participação social $(0,0001<\mathrm{p}<0,05)$, considerando o escore total, subescalas e a maioria das áreas do LIFE-H 3.1-Brasil (Tabela 3). Em indivíduos com presença de comprometimento motor do membro superior $(\mathrm{EFM} \leq 45)^{16}$, houve maior restrição, quando considerado o escore total, na subescala atividades diárias e nas áreas de nutrição, cuidados pessoais, moradia e responsabilidade.

Com relação aos fatores pessoais (Tabela 4), não houve diferença entre os grupos nos escores por área, subescalas e total, quando a idade e situação familiar foram consideradas. As mulheres apresentaram maior restrição na subescala papéis sociais e suas respectivas áreas (vida em comunidade, emprego e recreação). Indivíduos com tempo de evolução pós-AVE inferior a cinco anos apresentaram maior restrição na participação social nas subescalas atividades diárias e papéis sociais e na maioria das áreas, com exceção de relacionamentos interpessoais, nutrição e condicionamento físico (Tabela 4).

\section{DISCUSSÃO}

O objetivo deste estudo foi descrever e comparar o nível de restrição na participação social de indivíduos pósAVE na fase crônica, de acordo com variáveis de deficiências, limitações em mobilidade e fatores pessoais. Os resultados indicaram que, quando a amostra total foi considerada, foi observada restrição significativa apenas na área de recreação. A análise por grupos revelou que a restrição na participação social foi maior no grupo com tempo de evolução inferior a cinco anos e que apresentou deficiências e limitações de mobilidade. Considerando os fatores pessoais, as mulheres apresentaram maior restrição na subescala papéis sociais.

Considerando a amostra total, os resultados do presente estudo revelaram que a área de recreação foi a única que apresentou restrição significativa (escore $\leq 5,0)^{21}$. A área de recreação inclui participação em atividades esportivas ou recreativas, artísticas, culturais ou artesanais, turísticas e ao ar livre; ir a eventos esportivos e artísticos ou culturais e usar serviços de recreação da comunidade ${ }^{11}$. Pode-se observar que os hábitos de vida contemplados nessa área requerem boa habilidade de locomoção em ambiente comunitário, aspecto frequentemente reportado como comprometido após o AVE. No estudo de Robinson et al. ${ }^{5}, 22 \%$ dos indivíduos pós-AVE reportaram dificuldade de locomoção em ambiente comunitário interno (por exemplo, prédios públicos e estabelecimentos comerciais); $30 \%$ dificuldade para uso de transporte público e $72 \%$ dificuldade de locomoção em ambiente comunitário externo (calçadas ou ruas com declive ou terreno irregular). Assim, a dificuldade de locomoção em ambientes comunitários pode explicar a restrição significativa na área de recreação observada no presente estudo. Estudos anteriores demonstraram que, após o AVE, os indivíduos preferem a segurança do ambiente doméstico e substituem atividades sociais e de lazer ativo, como atividades esportivas, recreativas, artísticas ou culturais, por atividades individuais e de lazer passivo, o que inclui a leitura e assistir televisão $0^{22}$. Outro aspecto a ser destacado, que pode ter contribuído para a restrição nessa e em outras áreas da participação social, é a presença de sintomas depressivos, como reportado em estudo prévio, que observou restrição na participação em esportes, atividades recreativas e relações interpessoais em indivíduos pós-AVE com sintomas depressivos ${ }^{23}$.

$\mathrm{Na}$ análise por grupos, quanto às deficiências e limitações de mobilidade, os indivíduos, com presença de comprometimento motor de membros inferiores, marcha comunitária limitada e risco de quedas, apresentaram maior restrição na participação social, quando considerado o escore total do LIFE-H 3.1-Brasil, bem como as diversas áreas das subescalas atividades diárias e papeis sociais. Estes resultados estão de acordo com estudos prévios, que demonstraram a existência de associações positivas entre restrições em participação social e comprometimento motor do membro inferior ${ }^{4}$, menor velocidade de marcha ${ }^{4}$ emaior risco de quedas ${ }^{6}$ e indicam a relevância da mobilidade para participação em diversas áreas. Vários itens do LIFE-H 3.1-Brasil requerem diretamente habilidades de locomoção, como nas áreas de mobilidade e recreação, e também indiretamente, como nas áreas de nutrição e cuidados pessoais. Sendo assim, durante a reabilitação, a ênfase na recuperação de deficiências e redução das limitações referentes aos membros inferiores pode resultar em maiores níveis na participação social de indivíduos que sofreram um $\mathrm{AVE}^{4}$.

Semelhante às deficiências e limitações referentes aos membros inferiores, os indivíduos com sintomas depressivos apresentaram maior restrição na participação social, considerando o escore total, subescalas e a maioria das áreas. Este resultado é corroborado por estudo prévio, que demonstrou que indivíduos com sintomas depressivos na fase crônica de evolução pós-AVE apresentaram maior restrição na participação social2 ${ }^{23}$. Em indivíduos pós-AVE, a presença de sintomas depressivos foi associada a uma menor percepção de autoeficácia relacionada ao autocuidado ${ }^{24}$. O conceito de autoeficácia é descrito como a confiança do indivíduo em sua habilidade para desempenhar uma tarefa ou comportamento específico ${ }^{24}$. A redução da percepção de autoeficácia pode resultar em um menor engajamento em atividades cotidianas, e, consequentemente, em uma maior restrição na participação social. 
Faria-Fortini I, et al. Caracterização da participação social de indivíduos na fase crônica. Rev Ter Ocup Univ São Paulo. 2017 jan./abr.;28(1):71-8.

Tabela 3 - Média (DP) dos escores por área, subescalas e total do LIFE-H 3.1 Brasil, de acordo com as deficiências (sintomas depressivos e comprometimento motor) e limitações de mobilidade (velocidade de marcha e risco de quedas)

\begin{tabular}{|c|c|c|c|c|c|c|c|c|c|c|}
\hline \multirow{3}{*}{$\begin{array}{l}\text { LIFE-H } \\
\text { 3.1-Brasil }\end{array}$} & \multicolumn{6}{|c|}{ Deficiências } & \multicolumn{4}{|c|}{ Limitações de mobilidade } \\
\hline & \multicolumn{2}{|c|}{ Sintomas depressivos } & \multicolumn{2}{|c|}{$\begin{array}{c}\text { Comprometimento } \\
\text { motor - MS }\end{array}$} & \multicolumn{2}{|c|}{$\begin{array}{c}\text { Comprometimento } \\
\text { motor - MI }\end{array}$} & \multicolumn{2}{|c|}{$\begin{array}{c}\text { Velocidade } \\
\text { e marcha }(\mathbf{m} / \mathbf{s})\end{array}$} & \multicolumn{2}{|c|}{ Riscos de queda } \\
\hline & $\begin{array}{l}\mathrm{EDG}<6 \\
(\mathrm{n}=66)\end{array}$ & $\begin{array}{l}E D G \geq 6 \\
(n=40)\end{array}$ & $\begin{array}{l}\mathbf{E F M} \leq 45 \\
(\mathrm{n}=37)\end{array}$ & $\begin{array}{l}\mathrm{EFM}>45 \\
(\mathrm{n}=71)\end{array}$ & $\begin{array}{l}\mathrm{EFM} \leq 28 \\
(\mathrm{n}=58)\end{array}$ & $\begin{array}{l}\begin{array}{l}\text { EFM }>28 \\
(n=50)\end{array} \\
\end{array}$ & $\begin{array}{l}\leq 0,8 \\
(n=47) \\
\end{array}$ & $\begin{array}{l}>0,8 \\
(\mathrm{n}=60)\end{array}$ & $\begin{array}{l}\text { TUG }<15 s \\
(n=51)\end{array}$ & $\begin{array}{l}\text { TUG } \geq 15 \mathrm{~s} \\
(\mathrm{n}=55)\end{array}$ \\
\hline $\begin{array}{l}\text { Atividades } \\
\text { diárias }\end{array}$ & $7,9(1,1)^{* * * *}$ & $6,7(1,7)^{* * * * *}$ & $6,8(1,6)^{* *}$ & $7,7(1,3)^{* *}$ & $6,9(1,5) * * * *$ & $8,0(1,2)^{* * * *}$ & $6,9(1,5) * * * *$ & $7,9(1,1)^{* * * *}$ & $8,3(0,9)^{* * * *}$ & $6,7(1,4)^{* * * *}$ \\
\hline Nutrição & $7,9(1,7)^{* * * *}$ & $6,6(2,1)^{* * *}$ & $6,5(1,8) * * * *$ & $7,9(1,8)^{* * * * *}$ & $6,8(1,8)^{* * * *}$ & $8,1(1,8)^{* * * *}$ & $6,8(1,8)^{* *}$ & $7,9(1,8)^{* *}$ & $8,3(1,6)^{* * * *}$ & $6,6(1,8)^{* * * *}$ \\
\hline Condicionamento & $8,4(1,6) *$ & $7,7(1,9)^{*}$ & $8,1(1,9)$ & $8,0(1,7)$ & $7,8(1,8)$ & $8,4(1,7)$ & $7,5(1,9)^{* *}$ & $8,5(1,5)^{* *}$ & $8,7(1,5)^{* * *}$ & $7,6(1,8)^{* * *}$ \\
\hline Cuidados pessoais & $8,6(1,3)^{* * * * *}$ & $7,2(2,0)^{* * * * *}$ & $7,3(2,0)^{* *}$ & $8,5(1,4)^{* *}$ & $7,6(2,0)^{* *}$ & $8,6(1,2)^{* *}$ & $7,6(2,0)^{* *}$ & $8,5(1,3)^{* *}$ & $9,0(0,9)^{* * * *}$ & $7,4(1,8)^{* * * *}$ \\
\hline Comunicação & $8,4(1,3)^{* *}$ & $7,6(1,7)^{* *}$ & $7,7(1,7)$ & $8,2(1,4)$ & $7,9(1,5)$ & $8,1(1,5)$ & $7,8(1,5)$ & $8,2(1,4)$ & $8,4(1,4)^{*}$ & $7,7(1,5)^{*}$ \\
\hline Moradia & $7,3(1,6)^{* *}$ & $6,0(2,2) * *$ & $6,1(2,0)^{* *}$ & $7,2(1,8)^{* *}$ & $6,1(1,9)^{* * * *}$ & $7,7(1,6)^{* * * *}$ & $6,1(2,0)^{* * * *}$ & $7,4(1,6)^{* * * * *}$ & $7,9(1,4)^{* * * *}$ & $6,0(1,8)^{* * * *}$ \\
\hline Mobilidade & $6,2(2,2)^{* *}$ & $4,8(2,6)^{* *}$ & $5,2(2,4)$ & $6,0(2,4)$ & $4,8(2,3) * * * *$ & $6,7(2,1)^{* * * *}$ & $4,6(2,4) * * * *$ & $6,6(2,0)^{* * * *}$ & $6,9(2,0)^{* * * *}$ & $4,8(2,3) * * * *$ \\
\hline Papéis sociais & $7,4(1,9)^{* *}$ & $5,9(2,1)^{* *}$ & $6,2(2,4)$ & $7,1(2,0)$ & $6,2(2,2)^{* *}$ & $7,4(1,9)^{* *}$ & $5,9(2,0)^{* * * * *}$ & $7,5(2,0)^{* * * *}$ & $7,8(1,7)^{* * * *}$ & $5,9(2,1)^{* * * *}$ \\
\hline Responsabilidades & $8,2(1,9)^{* *}$ & $6,7(2,3)^{* *}$ & $6,9(2,6)^{*}$ & $7,9(2,0)^{*}$ & $7,1(2,5)^{*}$ & $8,1(1,7)^{*}$ & $6,9(2,4)^{* *}$ & $8,1(1,9)^{* *}$ & $8,4(1,7)^{* * * *}$ & $6,8(2,4) * * * *$ \\
\hline $\begin{array}{l}\text { Relacionamentos } \\
\text { interpessoais }\end{array}$ & $9,3(1,3)^{* * *}$ & $8,1(2,0)^{* * *}$ & $8,5(2,2)$ & $9,1(1,3)$ & $8,6(1,9)$ & $9,1(1,4)$ & $8,7(1,8)$ & $9,0(1,6)$ & $9,1(1,4)$ & $8,7(1,9)$ \\
\hline $\begin{array}{l}\text { Vida em } \\
\text { comunidade }\end{array}$ & $7,3(2,8)^{* *}$ & $5,6(3,1)^{* *}$ & $6,0(3,1)$ & $7,0(2,9)$ & $5,8(3,1)^{* *}$ & $7,6(2,6)^{* *}$ & $5,2(2,9) * * * *$ & $7,9(2,4)^{* * * *}$ & $8,1(2,2)^{* * * * *}$ & $5,5(3,0)^{* * * *}$ \\
\hline Emprego $(\mathrm{n}=91)$ & $6,8(3,4)$ & $6,0(3,6)$ & $5,8(3,6)$ & $6,7(3,5)$ & $6,5(3,6)$ & $6,3(3,6)$ & $5,6(3,6)^{*}$ & $7,1(3,4)^{*}$ & $7,5(3,2)^{* *}$ & $5,3(3,5)^{* *}$ \\
\hline Recreação (n=88) & $5,4(2,7)^{* *}$ & $3,8(2,8)^{* *}$ & $4,1(2,8)$ & $5,0(2,9)$ & $4,1(2,8)^{*}$ & $5,3(2,9)^{*}$ & $3,5(2,6)^{* *}$ & $5,4(2,8) * *$ & $5,7(3,0)^{* *}$ & $3,6(2,2)^{* *}$ \\
\hline Total & $7,7(1,3)^{* * * * *}$ & $6,4(1,8) * * * *$ & $6,5(1,9)^{* *}$ & $7,4(1,5)^{* *}$ & $6,6(1,7) * * * *$ & $7,7(1,4)^{* * * *}$ & $6,4(1,6) * * * *$ & $7,7(1,4)^{* * * * *}$ & $8,0(1,2) * * * *$ & $6,4(1,6)^{* * * *}$ \\
\hline
\end{tabular}

${ }^{*} \mathrm{p}<0,05 ; *{ }^{*} \mathrm{p}<0,01 ; * * \mathrm{p}<0,001 ; * * * \mathrm{p}<0,0001 ; \mathrm{EDG}=$ Escala de Depressão Geriátrica; EFM=Escala de Fugl Meyer; MS=membro superior; MI=membro inferior;

TUG $=$ Timed up and go test

Tabela 4 - Média (DP) dos escores por área, subescalas e total do LIFE-H 3.1 Brasil, de acordo com os fatores pessoais (sexo, idade, situação familiar e tempo de evolução)

\begin{tabular}{|c|c|c|c|c|c|c|c|c|}
\hline \multirow[b]{3}{*}{ LIFE-H 3.1-Brasil } & \multicolumn{8}{|c|}{ Fatores pessoais } \\
\hline & \multicolumn{2}{|c|}{ Sexo } & \multicolumn{2}{|c|}{ Idade } & \multicolumn{2}{|c|}{ Situação familiar } & \multicolumn{2}{|c|}{ Tempo de evolução } \\
\hline & $\begin{array}{l}\text { Homens } \\
(n=64)\end{array}$ & $\begin{array}{l}\text { Mulheres } \\
(n=45)\end{array}$ & $\begin{array}{l}<60 \text { anos } \\
(\mathrm{n}=55)\end{array}$ & $\begin{array}{l}>60 \text { anos } \\
(n=54)\end{array}$ & $\begin{array}{l}\text { Vive sozinho } \\
(n=12)\end{array}$ & $\begin{array}{l}\text { Vive com } \\
\text { familiar } \\
(\mathrm{n}=97)\end{array}$ & $\begin{array}{l}<5 \text { anos } \\
(n=65)\end{array}$ & $\begin{array}{l}>5 \text { anos } \\
(n=44)\end{array}$ \\
\hline Atividades diárias & $7,5(1,4)$ & $7,3(1,5)$ & $7,5(1,5)$ & $7,4(1,4)$ & $7,5(1,7)$ & $7,4(1,4)$ & $7,1(1,6)^{* * *}$ & $7,9(1,0)^{* * *}$ \\
\hline Nutrição & $7,3(2,0)$ & $7,4(1,8)$ & $7,2(2,0)$ & $7,6(1,8)$ & $7,1(2,1)$ & $7,4(1,9)$ & $7,1(1,9)$ & $7,7(1,8)$ \\
\hline Condicionamento físico & $8,3(1,8)$ & $7,7(1,7)$ & $8,0(1,7)$ & $8,1(1,8)$ & $8,2(2,4)$ & $8,1(1,7)$ & $7,8(1,9)$ & $8,4(1,5)$ \\
\hline Cuidados pessoais & $8,1(1,7)$ & $7,9(1,8)$ & $8,0(1,7)$ & $8,0(1,8)$ & $8,8(1,6)$ & $8,0(1,8)$ & $7,6(2,0)^{* * * *}$ & $8,7(1,1)^{* * * *}$ \\
\hline Comunicação & $8,2(1,5)$ & $7,8(1,4)$ & $8,2(1,4)$ & $7,9(1,6)$ & $7,6(2,3)$ & $8,1(1,4)$ & $7,7(1,6)^{*}$ & $8,5(1,2)^{*}$ \\
\hline Moradia & $6,8(1,9)$ & $6,8(2,1)$ & $6,9(1,9)$ & $6,7(2,0)$ & $6,8(1,4)$ & $6,8(2,0)$ & $6,5(2,1)^{*}$ & $7,3(1,5)^{*}$ \\
\hline Mobilidade & $5,8(2,2)$ & $5,6(2,7)$ & $5,8(2,5)$ & $5,5(2,3)$ & $5,7(2,9)$ & $5,7(2,4)$ & $5,3(2,5)^{*}$ & $6,3(2,2)^{*}$ \\
\hline Papéis sociais & $7,1(2,1)^{*}$ & $6,2(2,2)^{*}$ & $6,9(2,1)$ & $6,6(2,2)$ & $6,7(2,2)$ & $6,7(2,1)$ & $6,1(2,3)^{* * * *}$ & $7,6(1,7)^{* * * *}$ \\
\hline Responsabilidades & $7,7(2,3)$ & $7,3(2,1)$ & $7,8(2,1)$ & $7,3(2,3)$ & $7,6(2,6)$ & $7,5(2,2)$ & $7,1(2,3)^{*}$ & $8,2(2,0)^{*}$ \\
\hline $\begin{array}{l}\text { Relacionamentos } \\
\text { interpessoais }\end{array}$ & $8,8(1,8)$ & $9,0(1,4)$ & $8,6(1,8)$ & $9,2(1,5)$ & $7,9(2,8)$ & $9,0(1,5)$ & $8,8(1,9)$ & $9,1(1,3)$ \\
\hline Vida em comunidade & $7,2(2,8)^{*}$ & $5,9(3,2)^{*}$ & $7,0(2,9)$ & $6,2(3,1)$ & $7,1(3,1)$ & $6,5(3,0)$ & $5,9(3,2)^{*}$ & $7,7(2,5)^{*}$ \\
\hline Emprego $(\mathrm{n}=91)$ & $7,2(3,3)^{*}$ & $5,3(3,7)^{*}$ & $6,4(3,5)$ & $6,3(3,7)$ & $5,0(3,3)$ & $6,5(3,6)$ & $5,5(3,7)^{* *}$ & $7,4(3,2)^{* *}$ \\
\hline Recreação (n=88) & $5,3(2,8)^{*}$ & $3,7(2,9)^{*}$ & $4,8(2,9)$ & $4,4(2,9)$ & $4,3(2,4)$ & $4,7(3,0)$ & $3,8(2,8)^{* *}$ & $5,6(2,7)^{* *}$ \\
\hline Total & $7,3(1,6)$ & $6,8(1,7)$ & $7,2(1,7)$ & $7,0(1,7)$ & $7,2(1,8)$ & $7,1(1,7)$ & $6,7(1,8)^{* * * *}$ & $7,8(1,2)^{* * * *}$ \\
\hline
\end{tabular}

${ }^{*} \mathrm{p}<0,05 ;{ }^{* *} \mathrm{p}<0,01 ;{ }^{* * *} \mathrm{p}<0,001 ;{ }^{* * * *} \mathrm{p}<0,0001 ; \mathrm{DP}=$ desvio padrão 
O grupo com presença de comprometimento da função motora do membro superior também apresentou maior restrição na participação social, considerando e escore total e a subescala atividades diárias. Especificamente, quatro áreas apresentaram diferenças entre os grupos, sendo que todas elas contêm itens que requerem o uso funcional dos membros superiores. Aárea de nutrição inclui o preparo de refeições e manuseio de talheres; cuidados pessoais abrangem a realização de atividades de autocuidado, como higiene pessoal e vestir-se; moradia engloba a realização de tarefas de gerenciamento doméstico e uso de mobílias e equipamentos; e responsabilidades incluem o manuseio de dinheiro e uso de cartões bancários. A deficiência da função motora de membros superiores pode dificultar o alcance, preensão e transporte de objetos, reduzindo, desta forma, a participação em atividades cotidianas, que requerem o uso funcional dos membros superiores ${ }^{4}$. A existência de associações entre o comprometimento motor do membro superior e restrição na participação social já foi reportada em estudo prévio ${ }^{4}$. No entanto, deficiências dos membros superiores apresentaram associações mais fracas com a restrição na participação social do que as dos membros inferiores ${ }^{4}$, corroborando os resultados do presente estudo, já que o comprometimento motor do membro inferior impactou em um maior número de áreas da participação social.

Quanto aos fatores pessoais, destaca-se a influência do sexo na realização de hábitos de vida na subescala papéis sociais, sendo que mulheres apresentaram maior restrição nas áreas de vida em comunidade, emprego e recreação. Culturalmente, é atribuído a mulher a realização de tarefas de gerenciamento no ambiente doméstico ${ }^{8}$, o que pode restringir a realização de relevantes hábitos de vida em ambiente comunitário. Com relação à situação familiar e idade, não houve diferença entre os grupos, o que indica que a função e/ou a realização de atividades podem comprometer a participação social, mais do que a situação familiar e/ou as alterações fisiológicas decorrentes do processo de envelhecimento.
Por fim, observou-se que os indivíduos com tempo de evolução inferior a cinco anos apresentaram maior restrição na participação social. Uma possível explicação para este achado pode ser o desenvolvimento de estratégias de adaptação. Segundo Rozon e Rochette ${ }^{23}$, na fase crônica de evolução pós-AVE, pode ocorrer um aumento da participação social, quando comparado aos indivíduos na fase aguda. Este fato pode ser explicado pelo desenvolvimento de estratégias de adaptação pelo indivíduo, frente à sua nova condição de saúde. Já segundo Williams e Murray ${ }^{25}$, o processo de adaptação ocupacional pode ocorrer ao longo do tempo, sendo iniciado com um período de choque em decorrência da mudança súbita da habilidade física, seguido da vontade de superar as limitações e experimentar novas estratégias, e, finalmente, a aceitação de novas formas de realizar as atividades cotidianas. Este processo auxilia na redução da lacuna existente entre as habilidades do indivíduo e as demandas do ambiente após o $\mathrm{AVE}^{25}$. Desta forma, o maior período de convivência com as deficiências e limitações em atividades pode proporcionar uma maior capacidade de adaptação para realização de atividades cotidianas após o AVE.

\section{CONSIDERAÇÕS FINAIS}

Os resultados do presente estudo demonstraram que, na fase crônica após o AVE, há níveis diferenciados de participação social, sendo a restrição significativa na área de recreação. Além disso, dentre as deficiências, limitações de mobilidade e fatores pessoais analisados neste estudo, os aspectos mais envolvidos com restrições na participação social foram as deficiências relacionadas aos membros inferiores, limitação de mobilidade, sintomas depressivos e tempo de evolução pós-AVE. Estes aspectos devem ser considerados no planejamento de intervenções de reabilitação e elaboração de políticas públicas direcionadas a melhorar a participação social de indivíduos pós-AVE.

Responsabilidade de autoria: I Faria-Fortini, ML Basílio, JC Polese e KKP Menezes contribuíram com a concepção, o planejamento, a organização e o desenvolvimento do projeto; participaram de todo o seu desenvolvimento, desde a coleta dos dados e interpretação dos resultados até a elaboração do manuscrito, revisão crítica do seu conteúdo e aprovação da sua versão final. CDCM Faria, AA Scianni e LF Teixeira-Salmela colaboraram com o planejamento e desenvolvimento do projeto e participaram das etapas de tabulação dos dados, cálculos estatísticos e interpretação dos resultados, elaboração do manuscrito e revisão crítica do seu conteúdo e aprovação da sua versão final. 


\section{REFERÊNCIAS}

1. Brasil. Ministério da Saúde. Secretaria de Atenção à Saúde. Departamento de Ações Programáticas Estratégicas. Diretrizes de atenção à reabilitação da pessoa com acidente vascular cerebral. Brasília: Editora do Ministério da Saúde; 2013.

2. Mayo NE, Wood-Dauphinee S, Côté R, Durcan L, Carlton J. Activity, participation and quality of life 6 months poststroke. Arch Phys Med Rehabil. 2002;83(8):1035-42. doi: 10.1053/ apmr.2002.33984.

3. Organização Mundial de Saúde. Classificação Internacional de Funcionalidade, Incapacidade e Saúde. São Paulo: Universidade de São Paulo; 2003.

4. Desrosiers J, Malouin F, Bourbonnais D, Richards CL, Rochette A, Bravo G. Arm and leg impairments and disabilities after stroke rehabilitation: relation to handicap. Clin Rehabil. 2003;17(6):666-73. doi: 10.1191/0269215503cr662oa.

5. Robinson CA, Shumway-Cook A, Matsuda PN, Ciol MA. Understanding physical factors associated with participation in community ambulation following stroke. Disabil Rehabil. 2011;33(12):1033-42. doi: 10.3109/09638288.2010.520803.

6. Liu TW, NG SS, Kwong PW, Ng GY. Fear avoidance behavior, not walking endurance, predicts the community reintegration of community-dwelling stroke survivors. Arch Phys Med Rehabil. 2015;96(9):1684-90. doi: 10.1016/j. apmr.2015.05.005.

7. Chau JP, Thompson DR, Twinn S, Chang AM, Woo J. Determinants of participation restriction among community dwelling stroke survivors: a path analysis. BMC Neurol. 2009;7(9):49. doi: 10.1186/1471-2377-9-49.

8. Berzina G, Paanalahti M, Lundgren-Nilsson A, Sunnerhagen KS. Exploration of some personal factors with the International Classification of Functioning, Disability and Health core set for stroke. J Rehabil Med. 2013;45(7):609-15. doi: 10.2340/16501977-1171

9. Dhamoon MS, Moon YP, Paik MC, Boden-Albala B, Rundek T, Sacco RL, Elkind MS. Long-term functional recovery after first ischemic stroke: the Northern Manhattan Study. Stroke. 2009;40(8):2805-11. doi: 10.1161/ STROKEAHA.109.549576.

10. Bertolucci PHF, Brucki SMD, Campacci SR, Juliano Y. O mini-exame do estado mental em uma população geral. Arq Neuropsiquiatr. 1994;52(1):1-7.

11. Assumpção FSN, Faria-Fortini I, Magalhães LC, Basílio ML, Carvalho AC, Teixeira-Salmela LF. Propriedades de medida do LIFE-H 3.1-Brasil para avaliação da participação social de hemiparéticos. Rev Neurocienc. 2015;23(4):506-15. doi: 10.4181/RNC.2015.23.04.1082.

12. Almeida OP, Almeida AS. Confiabilidade da versão brasileira da escala de depressão em geriatria (GDS) versão reduzida. Arq Neuropsiquiatr. 1999;57(2-B):421-6.
13. Tang WK, Chan SS, Chiu HF, Wong KS, Kwok TC, Mok V, Ungvari GS. Can the geriatric depression scale detect poststroke depression in Chinese elderly? J Affect Disord. 2004;81(2):153-6. doi: 10.1016/S0165-0327(03)00163-0.

14. Maki T, Quagliato EMAB, Cacho EWA, Paz LPS, Nascimento NH, Inoue MMEA, Viana MA. Estudo de confiabilidade da aplicação da Escala de Fugl-Meyer no Brasil. Rev Bras Fisioter 2006;10(2):177-83.

15. Reynolds AM, Peters DM, Vendemia JM, Smith LP, Sweet RC, Baylis GC, Krotish D, Fritz SL. Neuronal injury in the motor cortex after chronic stroke and lower limb motor impairment: a voxel-based lesion symptom mapping study. Neural Regen Res. 2014;9(7):766-72. doi: 10.4103/1673-5374.131589.

16. Gebruers N, Truijen S, Engelborghs S, De Deyn PP. Predictive value of upper-limb accelerometry in acute stroke with hemiparesis. J Rehabil Res Dev. 2013;50(8):1099-106. doi: 10.1682/JRRD.2012.09.0166.

17. Faria CDCM, Teixeira-Salmela LF, Neto MG, Rodriguesde-Paula F. Performance-based tests in subjects with stroke: outcome scores, reliability and measurement errors. Clin Rehabil. 2012;26(5):460-9. doi: 10.1177/0269215511423849.

18. Bowden $\mathrm{MG}$, Balasubramanian $\mathrm{CK}$, Behrman $\mathrm{AL}$, Kautz SA. Validation of a speed-based classification system using quantitative measures of walking performance poststroke. Neurorehabil Neural Repair. 2008;22(6):672-5. doi: $10.1177 / 1545968308318837$

19. Persson CU, Hansson PO, Sunnerhagen KS. Clinical tests performed in acute stroke identify the risk of falling during the first year: Postural Stroke Study in Gothenburg (POSTGOT). J Rehabil Med. 2011;43(4): 348-53. doi: 10.2340/165019770677 .

20. Portney LG, Watkins MP. Foundations of clinical research: applications to practice. 3rd ed. New Jersey: Upper Saddle River; 2009.

21. Gagnon C, Mathieu J, Noreau L. Life habits in myotonic dystrophy type 1. J Rehabil Med. 2007;39(7):560-6. doi: 10.2340/16501977-0091.

22. O'Sullivan C, Chard G. An exploration of participation in leisure activities post-stroke. Aust Occup Ther J. 2010;57(3):159-66. doi: 10.1111/j.1440-1630.2009.00833.

23. Rozon J, Rochette A. Changes in life habits affected by mild stroke and their association with depressive symptoms. J Rehabil Med. 2015;47(6):495-501. doi: 10.2340/16501977-1959.

24. Robinson-Smith G, Johnston M, Allen J. Self-care self efficacy, quality of life, and depression after stroke. Arch Phys Med Rehabil. 2000;81(4):460-4. doi: 10.1053/mr.2000.3863.

25. Williams S, Murray C. The lived experience of older adults' occupational adaptation following a stroke. Aust Occup Ther J. 2013;60(1):39-47. doi: 10.1111/1440-1630.12004.

Recebido em: 08.06.16

Aceito em: 04.11.16 